\title{
A New Approach to Numerical Solution of Nonlinear Klein-Gordon Equation
}

\author{
Berna Bülbüll $^{1}$ and Mehmet Sezer ${ }^{2}$ \\ ${ }^{1}$ Department of Mathematics, Faculty of Science, Muğla University, 48000 Muğla, Turkey \\ ${ }^{2}$ Department of Mathematics, Faculty of Science, Celal Bayar University, 45000 Manisa, Turkey
}

Correspondence should be addressed to Mehmet Sezer; mehmet.sezer@cbu.edu.tr

Received 11 April 2013; Accepted 2 June 2013

Academic Editor: Ebrahim Momoniat

Copyright () 2013 B. Bülbül and M. Sezer. This is an open access article distributed under the Creative Commons Attribution License, which permits unrestricted use, distribution, and reproduction in any medium, provided the original work is properly cited.

A numerical method based on collocation points is developed to solve the nonlinear Klein-Gordon equations by using the Taylor matrix method. The method is applied to some test examples and the numerical results are compared with the exact solutions. The results reveal that the method is very effective, simple, and convenient. In addition, an error estimation of proposed method is presented.

\section{Introduction}

Nonlinear phenomena, that occurs in many areas of scientific such as solid state physics, plasma physics, fluid dynamics, mathematical biology and chemical kinematics, can be modeled by partial differential equations. The KleinGordon equation is an important class of partial differential equations and arises in relativistic quantum mechanics and field theory, which is great importance for the high energy physicist [1], and is used to model many different phenomena, including the propagation of dislocations in crystals and the behavior of elementary particles. On the other hand, the one-dimensional Klein-Gordon equation is given by partial differential equation [2]

$$
\frac{\partial^{2} u}{\partial t^{2}}-k^{2} \frac{\partial^{2} u}{\partial x^{2}}+h(u)=g(x, t) .
$$

In the present work we are dealing with the numerical approximation of the following nonlinear Klein-Gordon equation:

$$
\begin{aligned}
\frac{\partial^{2} u}{\partial t^{2}}- & k^{2} \frac{\partial^{2} u}{\partial x^{2}}+\beta_{1} u+\cdots+\beta_{n} u^{n} \\
& =g(x, t) \quad x \in[a, b], t \in[0, T],
\end{aligned}
$$

subject to initial conditions

$$
u(x, 0)=f(x), \quad \frac{\partial u(x, 0)}{\partial t}=m(x), \quad x \in[a, b],
$$

where $\beta_{i}(i=1, \ldots, n), k$ are physical constants and $g(x, t)$ is the time varying external input. Specially a non-zero righthand side in the KGE naturally arises in the context when including for instance quantum gravitational effects into the model.

KGE and the various other forms of the nonlinear KGE are all well studied in various papers. The equation has attracted much attention in studying solitons and soliton perturbation theory [3-6]. Biswas et al. studied the adiabatic dynamics of topological as well as the non-topological solitons in presence of perturbation terms [7-10]. In order to obtain the exact and numerical solutions of the nonlinear KGEs, a number of methods have been proposed such as the modified decomposition method [11], the symplectic finite difference approximations method [12], the numerical scheme based on the collocation method [2], the variational iteration method [13, 14], the finite element method [15], the cubic B-spline collocation method [16], the finite difference method [17], the decomposition method [18], Exp-function method $[19,20]$, the homotopy perturbation method [21], the tanh method [22] and the Jacobi elliptic function method 
[23], the Soliton solution [24-28], the stationary solutions [29] and the traveling wave solutions [30].

Since the beginning of 1994, Taylor matrix and collocation methods have been to solve linear and nonlinear ordinary differential equations, and difference equations used by Sezer et al. [7, 31-36]. In this work, the mentioned Taylor methods are developed and applied to Klein-Gordon equation (2) with the initial conditions (3), and the solution is expressed in the truncated double Taylor series

$$
u(x, t)=\sum_{r=0}^{N} \sum_{s=0}^{N} a_{r, s} x^{r} t^{s}, \quad a_{r, s}=\frac{1}{r ! \cdot s !} u^{(r, s)}(0,0),
$$

where $a_{r, s}$, for $r, s=0, \ldots, N$ are the coefficients to be determined. In this study we use the collocation points defined by

$$
\begin{array}{r}
x_{k}=a+k h, \quad t_{l}=l h ; \quad h=\frac{b-a}{N}, \\
k, l=0,1, \ldots, N .
\end{array}
$$

\section{Fundamental Relations}

In the numerical solution of (2) with the presented Taylor method, it is necessary to evaluate the Taylor coefficients of the unknown function. For convenience, the relation (4) can be written in the matrix form

$$
\begin{aligned}
u(x, t) & =\mathbf{X}_{1}(x, t) \mathbf{A}_{1}, \\
u^{i}(x, t) & =\mathbf{X}_{i}(x, t) \mathbf{A}_{i}, \quad i=2, \ldots, n,
\end{aligned}
$$

where $\mathbf{X}_{i}(x, t)$ and $\mathbf{A}_{i}$ are $1 \times(N+1)^{2^{i}}$ and $(N+1)^{2^{i}} \times 1$ matrices, respectively, and defined as follows:

$$
\begin{aligned}
& \mathbf{X}_{\mathbf{1}}(x, t)=\left[\begin{array}{llllll}
x^{0} t^{0} & x^{0} t^{1} \ldots x^{0} t^{N} & x^{1} t^{0} & x^{1} t^{1} \ldots x^{1} t^{N} \ldots
\end{array}\right. \\
& \left.x^{N} t^{0} x^{N} t^{1} \ldots x^{N} t^{N}\right] \\
& \mathbf{X}_{i}(x, t)=\left[\begin{array}{lllll}
\mathbf{X}_{0,0}^{(i)}(x, t) & \mathbf{X}_{0,1}^{(i)}(x, t) & \ldots & \mathbf{X}_{0, N}^{(i)}(x, t)
\end{array}\right. \\
& \mathbf{X}_{1,0}^{(i)}(x, t) \mathbf{X}_{1,1}^{(i)}(x, t) \ldots \mathbf{X}^{(i)}{ }_{1, N}(x, t) \ldots \\
& \left.\mathbf{X}_{N, 0}^{(i)}(x, t) \quad \mathbf{X}^{(i)}{ }_{N, 1}(x, t) \ldots \mathbf{X}^{(i)}{ }_{N, N}(x, t)\right],
\end{aligned}
$$

so that

$$
\begin{aligned}
& \mathbf{X}_{p, q}^{(i)}(x, t)=x^{p} t^{q} \mathbf{X}_{i-1}(x, t) ; \quad i=2, \ldots, n, \\
& p, q=0,1, \ldots, N \\
& \mathbf{A}_{1}=\left[\begin{array}{llllll}
a_{0,0} & a_{0,1} & \ldots & a_{0, N} & a_{1,0} & a_{1,1}
\end{array} \ldots\right. \\
& \left.a_{1, N} \ldots a_{N, 0} \quad a_{N, 1} \ldots a_{N, N}\right]^{T}, \\
& \mathbf{A}_{i}=\left[\begin{array}{llllll}
\mathbf{A}^{(i)}{ }_{0,0} & \mathbf{A}^{(i)}{ }_{0,1} & \ldots & \mathbf{A}^{(i)}{ }_{0, N} & \mathbf{A}^{(i)}{ }_{1,0} & \mathbf{A}^{(i)}{ }_{1,1} \ldots
\end{array}\right. \\
& \left.\mathbf{A}^{(i)}{ }_{1, N} \ldots \mathbf{A}_{N, 0}^{(i)} \mathbf{A}_{N, 1}^{(i)} \ldots \mathbf{A}_{N, N}^{(i)}\right]^{T},
\end{aligned}
$$

where

$$
\mathbf{A}_{p, q}^{(i)}=a_{p, q} \mathbf{A}_{i-1}, \quad i=2, \ldots, n, p, q=0,1, \ldots, N .
$$

On the other hand, the relation between the matrix $\mathbf{X}_{1}(x, t)$ and its derivatives $\left(\mathbf{X}_{1}(x, t)\right)^{(m, n)}$ is

$$
\left(\mathbf{X}_{1}(x, t)\right)^{(m, n)}=\mathbf{X}_{1}(x, t)(\overline{\mathbf{B}})^{m}(\widetilde{\mathbf{B}})^{n},
$$

where

$$
\begin{aligned}
\mathbf{B} & =\left[\begin{array}{ccccc}
0 & 1 & 0 & \cdots & 0 \\
0 & 0 & 2 & \cdots & 0 \\
\vdots & \vdots & \vdots & \ddots & \vdots \\
0 & 0 & 0 & \cdots & N \\
0 & 0 & 0 & \cdots & 0
\end{array}\right]_{(N+1) \times(N+1)}, \\
\overline{\mathbf{B}} & =\left[\begin{array}{ccccc}
0 & \mathbf{I} & 0 & \cdots & 0 \\
0 & 0 & 2 \mathbf{I} & \cdots & 0 \\
\vdots & \vdots & \vdots & \ddots & \vdots \\
0 & 0 & 0 & \cdots & N \mathbf{I} \\
0 & 0 & 0 & \cdots & 0
\end{array}\right]_{(N+1)^{2} \times(N+1)^{2}} \\
\widetilde{\mathbf{B}} & =\left[\begin{array}{cccc}
\mathbf{B} & 0 & \cdots & 0 \\
0 & \mathbf{B} & \cdots & 0 \\
\vdots & \vdots & \ddots & \vdots \\
0 & 0 & \cdots & \mathbf{B}
\end{array}\right]_{(N+1)^{2} \times(N+1)^{2}},
\end{aligned}
$$

where $\mathbf{I}$ is the $(N+1) \times(N+1)$ identity matrix. Using the relations (6) and (10) we have

$$
u^{(m, n)}(x, t)=\left(\mathbf{X}_{1}(x, t)\right)^{(m, n)} \mathbf{A}=\mathbf{X}_{1}(x, t)(\overline{\mathbf{B}})^{m}(\widetilde{\mathbf{B}})^{n} \mathbf{A}_{1} .
$$

\section{Method of Solution}

The technique is to assume that the unknown function has an expansion in the form (4), and to attempt to determine values for the coefficients $\left\{a_{r, s}\right\}$ such that the required differential equation and other conditions are satisfied. To obtain numerical approximation to Klein-Gordon equation under the given conditions, we can reduce (2) and (3) to the matrix equations as follows:

$$
\begin{aligned}
& \mathbf{X}_{1}(x, t)\left\{(\widetilde{\mathbf{B}})^{2}-k^{2}(\overline{\mathbf{B}})^{2}\right\} \mathbf{A}_{1}+\beta_{1} \mathbf{X}_{1}(x, t) \mathbf{A}_{1} \\
& +\beta_{2} \mathbf{X}_{2}(x, t) \mathbf{A}_{2}+\cdots+\beta_{n} \mathbf{X}_{n}(x, t) \mathbf{A}_{n}=g(x, t),
\end{aligned}
$$

or briefly

$$
\mathbf{W}_{1}(x, t) \mathbf{A}_{1}+\mathbf{W}_{2}(x, t) \mathbf{A}_{2}+\cdots+\mathbf{W}_{n}(x, t) \mathbf{A}_{n}=g(x, t),
$$

where

$$
\begin{gathered}
\mathbf{W}_{1}(x, t)=\mathbf{X}_{1}(x, t)\left\{(\widetilde{\mathbf{B}})^{2}-k^{2}(\overline{\mathbf{B}})^{2}\right\}+\beta_{1} \mathbf{X}_{1}(x, t), \\
\mathbf{W}_{i}(x, t)=\beta_{i} \mathbf{X}_{i}(x, t), \quad i=2, \ldots, n .
\end{gathered}
$$


By substituting the collocation points defined by (5) into (14) we have

$$
\begin{gathered}
\mathbf{W}_{1}\left(x_{k}, t_{l}\right) \mathbf{A}_{1}+\mathbf{W}_{2}\left(x_{k}, t_{l}\right) \mathbf{A}_{2}+\cdots+\mathbf{W}_{n}\left(x_{k}, t_{l}\right) \mathbf{A}_{n} \\
=g\left(x_{k}, t_{l}\right) ; \quad k, l=0,1, \ldots, N
\end{gathered}
$$

or in the compact form

$$
\widetilde{\mathbf{W}}_{1} \mathbf{A}_{1}+\widetilde{\mathbf{W}}_{2} \mathbf{A}_{2}+\cdots+\widetilde{\mathbf{W}}_{n} \mathbf{A}_{n}=\mathbf{G},
$$

where

$$
\begin{aligned}
& \widetilde{\mathbf{w}}_{j}=\left[\begin{array}{c}
\widetilde{\mathbf{w}}_{0}^{j} \\
\widetilde{\mathbf{w}}_{1}^{j} \\
\vdots \\
\widetilde{\mathbf{w}}_{N}^{j}
\end{array}\right], \quad \widetilde{\mathbf{w}}_{i}^{j}=\left[\begin{array}{c}
\mathbf{w}_{i}\left(x_{0}, t_{i}\right) \\
\mathbf{W}_{i}\left(x_{1}, t_{i}\right) \\
\vdots \\
\mathbf{W}_{i}\left(x_{N}, t_{i}\right)
\end{array}\right], \quad \mathbf{G}=\left[\begin{array}{c}
\mathbf{g}_{0} \\
\mathbf{g}_{1} \\
\vdots \\
\mathbf{g}_{N}
\end{array}\right], \\
& \mathbf{g}_{i}=\left[\begin{array}{c}
g\left(x_{0}, t_{i}\right) \\
g\left(x_{1}, t_{i}\right) \\
\vdots \\
g\left(x_{N}, t_{i}\right)
\end{array}\right], \quad i=0, \ldots, N, j=1,2, \ldots, n .
\end{aligned}
$$

We can use the following formulas to construct the matrix representation of initial conditions (3):

$$
u(x, t)=\mathbf{X}(x) \mathbf{Q}(t) \mathbf{A}_{1}, \quad u_{t}(x, t)=\mathbf{X}(x) \mathbf{Q}(t) \widetilde{\mathbf{B}} \mathbf{A}_{1},
$$

where

$$
\begin{aligned}
& \mathbf{X}(x)=\left[\begin{array}{lllll}
1 & x & x^{2} & \ldots & x^{N}
\end{array}\right], \quad \mathbf{T}(t)=\left[\begin{array}{lllll}
1 & t & t^{2} & \ldots & t^{N}
\end{array}\right], \\
& \mathbf{Q}(t)=\left[\begin{array}{cccc}
\mathbf{T}(t) & 0 & \cdots & 0 \\
0 & \mathbf{T}(t) & \cdots & 0 \\
\vdots & \vdots & \ddots & \vdots \\
0 & 0 & \cdots & \mathbf{T}(t)
\end{array}\right] \text {. }
\end{aligned}
$$

The matrix representations of nonhomogeneous terms of (3) can be written in the forms

$$
\begin{gathered}
f(x)=\mathbf{X}(x) \mathbf{F}, \quad \mathbf{F}=\left[\begin{array}{llll}
f_{0} & f_{1} & \ldots & f_{N}
\end{array}\right]^{T}, \\
f_{n}=\frac{f^{(n)}(0)}{n !} ; \quad n=0,1, \ldots, N, \\
m(x)=\mathbf{X}(x) \mathbf{M}, \quad \mathbf{M}=\left[\begin{array}{llll}
m_{0} & m_{1} & \ldots & m_{N}
\end{array}\right]^{T}, \\
m_{n}=\frac{m^{(n)}(0)}{n !} ; \quad n=0,1, \ldots, N .
\end{gathered}
$$

By substituting relations (21) into (3) and then simplifying the result, we get the matrix forms of conditions as

$$
\mathbf{Q}(0) \mathbf{A}_{1}=\mathbf{F}, \quad \mathbf{Q}(0) \widetilde{\mathbf{B}} \mathbf{A}_{1}=\mathbf{M} \text {. }
$$

TABLE 1: $L_{\infty}, L_{2}$, and RMS errors of Example 2 for $N=4$ and $h=$ 0.1 .

\begin{tabular}{lccc}
\hline$t$ & $L_{\infty}$-error & $L_{2}$-error & RMS-error \\
\hline 0 & 0 & 0 & 0 \\
0.1 & $4.9593 E-8$ & $9.6138 E-6$ & $2.8986 E-6$ \\
0.2 & $5.6542 E-7$ & $5.4883 E-6$ & $1.6532 E-6$ \\
0.3 & $1.8493 E-6$ & $1.1933 E-5$ & $3.5979 E-6$ \\
0.4 & $3.2841 E-6$ & $1.5829 E-5$ & $4.7726 E-6$ \\
0.5 & $3.8373 E-6$ & $1.4683 E-5$ & $4.4271 E-6$ \\
0.6 & $4.6197 E-6$ & $1.4714 E-5$ & $4.4366 E-6$ \\
0.7 & $1.4141 E-5$ & $3.9360 E-5$ & $1.1867 E-5$ \\
0.8 & $5.6879 E-5$ & $1.3952 E-4$ & $4.2069 E-5$ \\
0.9 & $1.8571 E-4$ & $4.0542 E-4$ & $1.2224 E-4$ \\
1 & $2.2334 E-4$ & $9.7993 E-4$ & $2.9546 E-4$ \\
\hline
\end{tabular}

To obtain the solution under the conditions, we get

$$
\begin{gathered}
\overline{\mathbf{W}}_{1}=\left[\begin{array}{c}
\widetilde{\mathbf{w}}_{0}^{j} \\
\widetilde{\mathbf{w}}_{1}^{j} \\
\vdots \\
\widetilde{\mathbf{w}}_{N-2}^{j} \\
\mathbf{Q}(0) \\
\mathbf{Q}(0) \widetilde{\mathbf{B}}
\end{array}\right], \quad \overline{\mathbf{W}}_{j}=\left[\begin{array}{c}
\widetilde{\mathbf{w}}_{0}^{j} \\
\widetilde{\mathbf{w}}_{1}^{j} \\
\vdots \\
\widetilde{\mathbf{w}}_{N-2}^{j} \\
0 \\
0
\end{array}\right], \quad(j=2, \ldots, n), \\
\overline{\mathbf{G}}=\left[\begin{array}{c}
\mathbf{g}_{0} \\
\mathbf{g}_{1} \\
\vdots \\
\mathbf{g}_{N-2} \\
\mathbf{F} \\
\mathbf{M}
\end{array}\right] .
\end{gathered}
$$

By solving the following nonlinear system:

$$
\overline{\mathbf{W}}_{1} \mathbf{A}_{1}+\overline{\mathbf{W}}_{2} \mathbf{A}_{2}+\cdots+\overline{\mathbf{W}}_{n} \mathbf{A}_{n}=\overline{\mathbf{G}}
$$

the unknown Taylor coefficients $a_{r, s}$ are determined and substituted in (4); thus we get the Taylor polynomial solution

$$
u(x, t)=\sum_{r=0}^{N} \sum_{s=0}^{N} a_{r, s} x^{r} t^{s}
$$

\section{Accuracy of Solution and Error Analysis}

We can easily check the accuracy of the solution. Since the truncated Taylor series (4) is an approximate solution of (2), when the function $u_{N}(x, t)$ and its derivatives are substituted 
TABLE 2: Absolute errors of Example 3 for $N=4$.

\begin{tabular}{|c|c|c|c|c|c|c|}
\hline \multirow{2}{*}{$\begin{array}{l}t \\
x_{i}\end{array}$} & \multicolumn{2}{|c|}{$t=0.001$} & \multicolumn{2}{|c|}{$t=0.01$} & \multicolumn{2}{|c|}{$t=0.1$} \\
\hline & $c=0.01$ & $c=0.05$ & $c=0.01$ & $c=0.05$ & $c=0.01$ & $c=0.05$ \\
\hline 0.1 & $5.13 E-9$ & $5.19 E-9$ & $6.18 E-7$ & $5.20 E-7$ & $5.88 E-5$ & $5.91 E-5$ \\
\hline 0.2 & $9.58 E-6$ & $9.61 E-6$ & $9.39 E-6$ & $9.42 E-6$ & $1.63 E-5$ & $1.63 E-5$ \\
\hline 0.3 & $3.67 E-6$ & $3.68 E-6$ & $3.67 E-6$ & $3.68 E-6$ & $1.20 E-6$ & $2.91 E-6$ \\
\hline 0.4 & $2.68 E-6$ & $2.69 E-6$ & $2.61 E-6$ & $2.62 E-6$ & $2.42 E-6$ & $1.23 E-6$ \\
\hline 0.4 & $3.66 E-6$ & $3.67 E-6$ & $3.60 E-6$ & $3.61 E-6$ & $3.69 E-6$ & $2.47 E-6$ \\
\hline 0.5 & $3.91 E-11$ & $4.61 E-6$ & $2.02 E-9$ & $2.19 E-6$ & $5.18 E-6$ & $3.76 E-6$ \\
\hline 0.6 & $3.84 E-6$ & $3.86 E-6$ & $3.78 E-6$ & $3.79 E-6$ & $6.85 E-6$ & $5.28 E-6$ \\
\hline 0.7 & $2.96 E-6$ & $2.97 E-6$ & $2.87 E-6$ & $2.88 E-6$ & $4.65 E-6$ & $7.00 E-6$ \\
\hline 0.8 & $4.26 E-6$ & $4.27 E-6$ & $1.14 E-6$ & $1.12 E-5$ & $1.39 E-5$ & $1.37 E-5$ \\
\hline 0.9 & $1.16 E-5$ & $1.17 E-5$ & $4.27 E-6$ & $3.63 E-6$ & $7.51 E-5$ & $7.36 E-5$ \\
\hline 1 & $6.90 E-9$ & $9.88 E-5$ & $1.16 E-7$ & $2.68 E-6$ & $1.39 E-5$ & $1.44 E-5$ \\
\hline
\end{tabular}

TABLE 3: Error analysis for Example 4.

\begin{tabular}{lc}
\hline$x_{i}$ & $E_{N}\left(x_{i}, 0.5\right)$ \\
\hline 0 & 0 \\
0.1 & $4.14 E-4$ \\
0.2 & $3.39 E-4$ \\
0.3 & $3.54 E-4$ \\
0.4 & $1.19 E-4$ \\
0.5 & $7.83 E-4$ \\
0.6 & $6.13 E-10$ \\
0.7 & $1.41 E-4$ \\
0.8 & $7.68 E-4$ \\
0.9 & $2.71 E-4$ \\
1 & $8.41 E-4$ \\
\hline
\end{tabular}

in (2), the resulting equation must be satisfied approximately; that is, for $x=x_{p}, t=t_{q} \in[a, b] \times[0, T], p, q=0,1,2 \ldots$

$$
\begin{aligned}
E_{N}\left(x_{p}, t_{q}\right)= & \mid \frac{\partial^{2} u\left(x_{p}, t_{q}\right)}{\partial t^{2}}-k^{2} \frac{\partial^{2} u\left(x_{p}, t_{q}\right)}{\partial x^{2}}+\beta_{1} u\left(x_{p}, t_{q}\right) \\
& +\cdots+\beta_{n} u^{n}\left(x_{p}, t_{q}\right)-g\left(x_{p}, t_{q}\right) \mid \cong 0
\end{aligned}
$$

where

$$
E_{N}\left(x_{p}, t_{q}\right) \leq 10^{-k_{p q}}, \quad\left(k_{p q} \text { is any positive integer }\right) \text {. }
$$

If $\max 10^{-k_{p q}}=10^{-k}$ ( $k$ positive integer) is prescribed, then the truncation limit $N$ is increased until difference $E\left(x_{p}, t_{q}\right)$ at each of the points becomes smaller than the prescribed $10^{-k}$. On the other hand we use different error norms for measuring errors. These error norms are defined as follows:
(1) $L_{2}-$ Error $=\left(\sum_{i=0}^{n}\left(e_{i}\right)^{2}\right)^{1 / 2}$,
(2) $L_{\infty}-$ Error $=\operatorname{Max}\left(e_{i}\right), 0 \leq i \leq n$,
(3) RMS - Error $=\sqrt{\sum_{i=0}^{n} e_{i}^{2} /(n+1)}$,

where $e_{i}=u\left(x_{i}, \tau\right)-\widehat{u}\left(x_{i}, \tau\right)\left(x_{i}=a+i h, h=(b-a) / n\right)$; also $u$ and $\widehat{u}$ are the exact and approximate solutions of the problem, respectively, and $\tau$ is an arbitrary time $t$ in $[0, T]$.

\section{Illustrative Examples}

In this section, several numerical examples are given to illustrate the properties of the method and all of them were performed on the computer using a program written in Maple v9.

Example 1. Consider the nonlinear Klein-Gordon equation [13]

$$
u_{t t}-u_{x x}+u^{2}=6 x t\left(x^{2}-t^{2}\right)+x^{6} t^{6}
$$

with initial conditions

$$
u(x, 0)=0, \quad u_{t}(x, 0)=0 .
$$

Following the procedures in Section 2 and by substituting the obtained coefficients in equation, the solution becomes

$$
u(x, t)=x^{3} t^{3}
$$

which is the exact solution.

Example 2. We next consider the nonlinear linear KleinGordon equation [2]

$$
u_{t t}-u_{x x}+u^{2}=-x \cos (t)+x^{2} \cos ^{2}(t),
$$

with initial conditions

$$
u(x, 0)=x, \quad u_{t}(x, 0)=0 .
$$

In Table 1, the $L_{2}, L_{\infty}$ errors and RMS of errors are obtained for different values of $t$. The graph of analytical and numerical functions for $N=4$ and the absolute error graph are given in Figure 1. It is seen that the linear terms in right hand side of (28) and (31) are forced terms. By adding these terms to the homogen equations, we are trying to find the effect of the force terms on homogen solution such a way that either they are in solution forms or other types of solutions. 


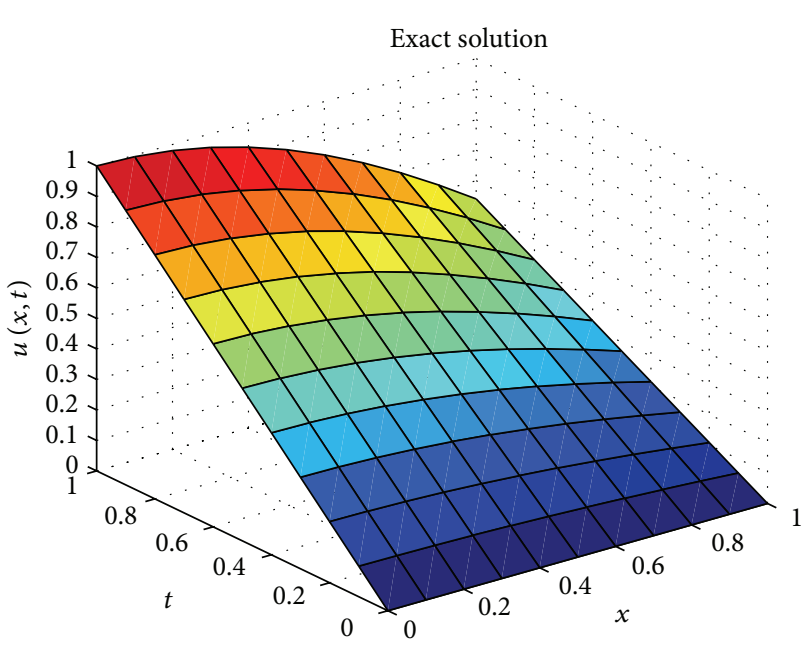

(a)

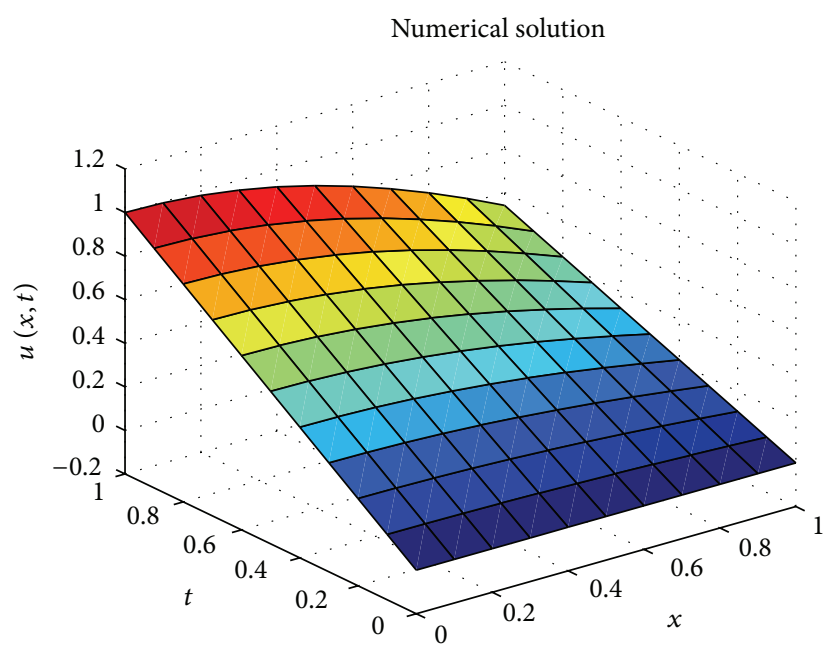

(b)

Absolute error

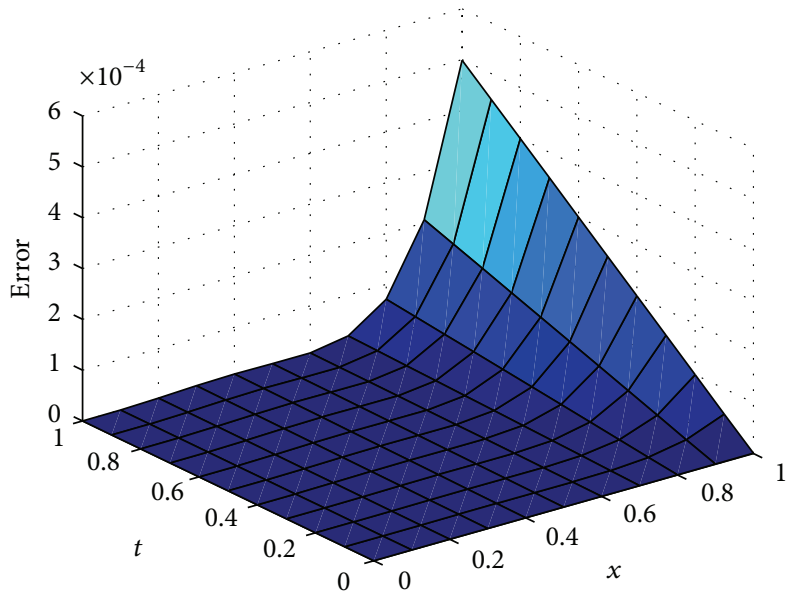

(c)

FIgURE 1: Exact solution, numerical solution, and absolute error of Example 2 for $N=4$

Example 3. In this example, we consider the nonlinear KleinGordon Equation (3) with cubic nonlinearity with constants $k^{2}=2.5, \beta_{1}=1, \beta_{3}=1.5$ and $\left.\beta_{i}=0, i \neq 1,3\right)$ in the interval $0 \leq x \leq 1$. The initial conditions are given by

$$
\begin{aligned}
& u(x, 0)=B \tan (K x), \quad 0 \leq x \leq 1, \\
& u_{t}(x, 0)=B c K \sec ^{2}(K x), \quad 0 \leq x \leq 1,
\end{aligned}
$$

and the exact solution by (3) is

$$
u(x, t)=B \tan (K(x+c t)), \quad 0 \leq x \leq 1,
$$

where

$$
B=\sqrt{\frac{\beta_{1}}{\beta_{3}}}, \quad K=\sqrt{\frac{-\beta_{1}}{2\left(k^{2}+c^{2}\right)}}, \quad g(x, t)=0 .
$$

The numerical solution for Example 3 is recorded for different time levels by utilizing the proposed method. At each given time level, absolute error is shown in Table 2.
Example 4. We finally close our analysis by studying the Klein-Gordon equation [14]

$$
u_{t t}-u_{x x}+\frac{3}{4} u-\frac{3}{2} u^{3}=0
$$

with initial conditions

$$
u(x, 0)=-\operatorname{sech}(x), \quad u_{t}(x, 0)=\frac{1}{2} \operatorname{sech}(x) \tan h(x) .
$$

Here the exact solution of equation is (see [21])

$$
u(x, t)=-\operatorname{sech}\left(x+\frac{1}{2} t\right)
$$

The solution of this nonlinear system is obtained for $N=6$. For numerical results, see Table 3 and Figure 2. 


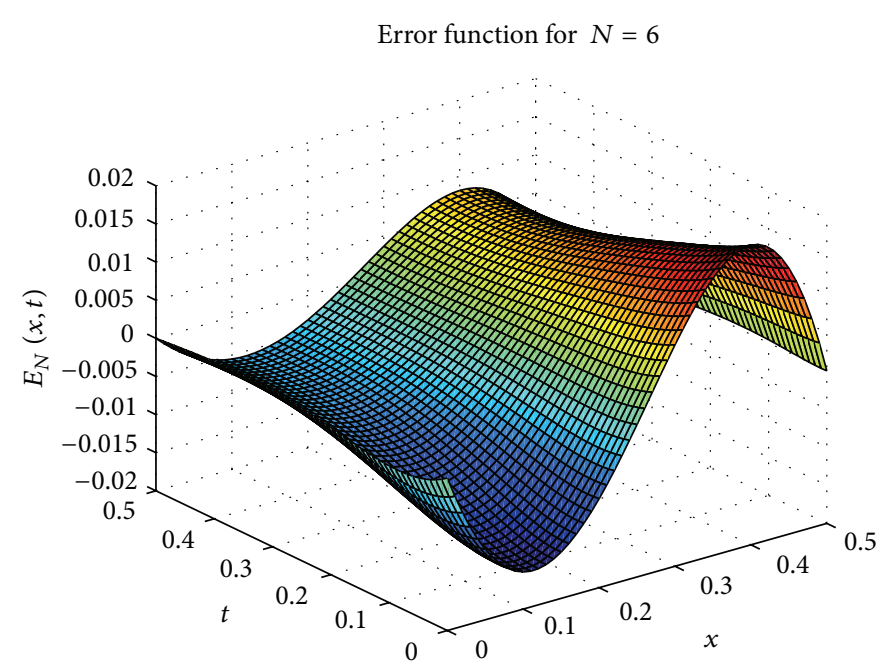

(a)

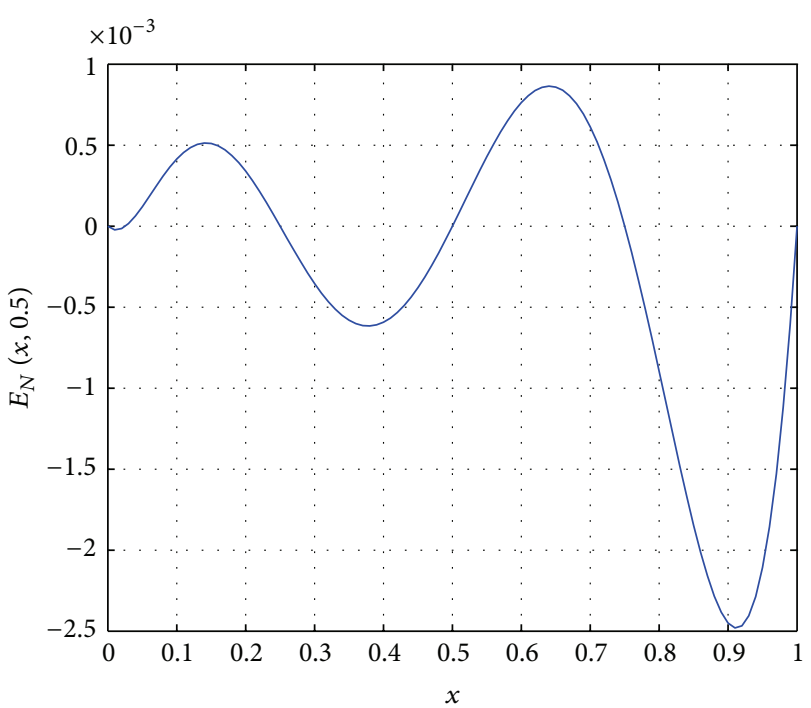

(b)

Figure 2: (a) Error function for Example 4. (b) Error function at $t=0.5$ for Example 4.

\section{Conclusion}

In this paper, a very simple but effective Taylor matrix method was proposed for the numerical solution nonlinear KleinGordon equation. One of the advantages of this method that the solution is expressed as a truncated Taylor series, then $u(x, t)$ can be easily evaluated for arbitrary values of $x$ and $t$ by using the computer program without any computational effort. From the given illustrative examples, it can be seen that the Taylor series approach can obtain very accurate and satisfactory results. An interesting feature of this method is that the analytical solution is obtained as demonstrated in Example 1 when the exact solution is polynomial. This method can be improved with new strategies to solve the other nonlinear equations.

\section{References}

[1] W. Greiner, Relativistic Quantum Mechanics-Wave Equations, Springer-Verlag, Berlin, Germany, 3rd edition, 2000.

[2] M. Dehghan and A. Shokri, "Numerical solution of the nonlinear Klein-Gordon equation using radial basis functions," Journal of Computational and Applied Mathematics, vol. 230, no. 2, pp. 400-410, 2009.

[3] A. Biswas, A. Yildirim, T. Hayat, O. M. Aldossary, and R. Sassaman, "Soliton perturbation theory for the generalized KleinGordon equation with full nonlinearity," Proceedings of the Romanian Academy A, vol. 13, no. 1, pp. 32-41, 2012.

[4] A. Biswas, C. Zony, and E. Zerrad, "Soliton perturbation theory for the quadratic nonlinear Klein-Gordon equation," Applied Mathematics and Computation, vol. 203, no. 1, pp. 153-156, 2008.

[5] A. Biswas, G. Ebadi, M. Fessak et al., "Solutions of the perturbed Klein-Gordon equations," Iranian Journal of Science and Technology A, vol. 36, no. 4, pp. 431-452, 2012.
[6] R. Sassaman and A. Biswas, "Soliton perturbation theory for phi-four model and nonlinear Klein-Gordon equations," Communications in Nonlinear Science and Numerical Simulation, vol. 14, no. 8, pp. 3239-3249, 2009.

[7] R. Sassaman and A. Biswas, "Topological and non-topological solitons of the generalized Klein-Gordon equations," Applied Mathematics and Computation, vol. 215, no. 1, pp. 212-220, 2009.

[8] R. Sassaman, A. Heidari, and A. Biswas, "Topological and nontopological solitons of nonlinear Klein-Gordon equations by He's semi-inverse variational principle," Journal of the Franklin Institute, vol. 347, no. 7, pp. 1148-1157, 2010.

[9] R. Sassaman, A. Heidari, F. Majid, E. Zerrad, and A. Biswas, "Topological and non-topological solitons of the generalized Klein-Gordon equations in 1+2 dimensions," Dynamics of Continuous, Discrete \& Impulsive Systems A, vol. 17, no. 2, pp. 275286, 2010.

[10] R. Sassaman and A. Biswas, "Topological and non-topological solitons of the Klein-Gordon equations in 1+2 dimensions," Nonlinear Dynamics, vol. 61, no. 1-2, pp. 23-28, 2010.

[11] A. Wazwaz, "The modified decomposition method for analytic treatment of differential equations," Applied Mathematics and Computation, vol. 173, no. 1, pp. 165-176, 2006.

[12] D. B. Duncan, "Symplectic finite difference approximations of the nonlinear Klein-Gordon equation," SIAM Journal on Numerical Analysis, vol. 34, no. 5, pp. 1742-1760, 1997.

[13] S. Abbasbandy, "Numerical solution of non-linear KleinGordon equations by variational iteration method," International Journal for Numerical Methods in Engineering, vol. 70, no. 7, pp. 876-881, 2007.

[14] E. Yusufoğlu, "The variational iteration method for studying the Klein-Gordon equation," Applied Mathematics Letters, vol. 21, no. 7, pp. 669-674, 2008.

[15] Q. Wang and D. Cheng, "Numerical solution of damped nonlinear Klein-Gordon equations using variational method and finite element approach," Applied Mathematics and Computation, vol. 162, no. 1, pp. 381-401, 2005. 
[16] J. Rashidinia, M. Ghasemi, and R. Jalilian, "Numerical solution of the nonlinear Klein-Gordon equation," Journal of Computational and Applied Mathematics, vol. 233, no. 8, pp. 1866-1878, 2010.

[17] H. Han and Z. Zhang, "An analysis of the finite-difference method for one-dimensional Klein-Gordon equation on unbounded domain," Applied Numerical Mathematics, vol. 59, no. 7, pp. 1568-1583, 2009.

[18] D. Kaya and S. M. El-Sayed, "A numerical solution of the Klein-Gordon equation and convergence of the decomposition method," Applied Mathematics and Computation, vol. 156, no. 2, pp. 341-353, 2004.

[19] A. Ebaid, "Exact solutions for the generalized Klein-Gordon equation via a transformation and Exp-function method and comparison with Adomian's method," Journal of Computational and Applied Mathematics, vol. 223, no. 1, pp. 278-290, 2009.

[20] T.-M. Wang and J.-M. Zhu, "New explicit solutions of the Klein-Gordon equation using the variational iteration method combined with the Exp-function method," Computers \& Mathematics with Applications, vol. 58, no. 11-12, pp. 2444-2448, 2009.

[21] Z. Odibat and S. Momani, "A reliable treatment of homotopy perturbation method for Klein-Gordon equations," Physics Letters A, vol. 365, no. 5-6, pp. 351-357, 2007.

[22] A.-M. Wazwaz, "Compactons, solitons and periodic solutions for variants of the KdV and the KP equations," Applied Mathematics and Computation, vol. 161, no. 2, pp. 561-575, 2005.

[23] S. Liu, Z. Fu, and S. Liu, "Periodic solutions for a class of coupled nonlinear partial differential equations," Physics Letters A, vol. 336 , no. 2-3, pp. 175-179, 2005.

[24] M. Song, Z. Liu, E. Zerrad, and A. Biswas, "Singular soliton solution and bifurcation analysis of Klein-Gordon equation with power law nonlinearity," Frontiers of Mathematics in China, vol. 8, no. 1, pp. 191-201, 2013.

[25] M. Song, Z. liu, E. Eerrad, and A. Biswas, "Singular solitons and bifurcation analysis of quadratic nonlinear klein-gordon equation," Applied Mathematics and Information Sciences, vol. 7, no. 4, pp. 1333-1340, 2013.

[26] R. Sassaman and A. Biswas, "Soliton solution of the generalized klein-gordon equation by semi-inverse variational principle," Mathematics in Engineering Science and Aerospace, vol. 2, no. 1, pp. 99-104, 2011.

[27] R. Sassaman and A. Biswas, "1-soliton solution of the perturbed klein-gordon equation," Physics Express, vol. 1, no. 1, pp. 9-14, 2011.

[28] R. Sassaman, M. Edwards, F. Majid, and A. Biswas, "1-soliton solution of the coupled nonlinear klein-gordon equations," Studies in Mathematical Sciences, vol. 1, no. 1, pp. 30-37, 2010.

[29] C. M. Khalique and A. Biswas, "Analysis of non-linear KleinGordon equations using Lie symmetry," Applied Mathematics Letters, vol. 23, no. 11, pp. 1397-1400, 2010.

[30] A. Biswas, C. M. Khalique, and A. R. Adem, "Traveling wave solutions of the nonlinear dispersive Klein-Gordon equations," Journal of King Saud University, vol. 24, no. 4, pp. 339-342, 2012.

[31] M. Sezer, "Taylor polynomial solutions of Volterra integral equations," International Journal of Mathematical Education in Science and Technology, vol. 25, no. 5, pp. 625-633, 1994.

[32] M. Gülsu and M. Sezer, "On the solution of the Riccati equation by the Taylor matrix method," Applied Mathematics and Computation, vol. 188, no. 2, pp. 446-449, 2007.

[33] M. Sezer and M. Gülsu, "A new polynomial approach for solving difference and Fredholm integro-difference equations with mixed argument," Applied Mathematics and Computation, vol. 171, no. 1, pp. 332-344, 2005.

[34] B. Bülbül and M. Sezer, "A Taylor matrix method for the solution of a two-dimensional linear hyperbolic equation," Applied Mathematics Letters, vol. 24, no. 10, pp. 1716-1720, 2011.

[35] B. Bülbül, M. Gülsu, and M. Sezer, "A new Taylor collocation method for nonlinear Fredholm-Volterra integro-differential equations," Numerical Methods for Partial Differential Equations, vol. 26, no. 5, pp. 1006-1020, 2010.

[36] B. Bülbül and M. Sezer, "Taylor polynomial solution of hyperbolic type partial differential equations with constant coefficients," International Journal of Computer Mathematics, vol. 88, no. 3, pp. 533-544, 2011. 


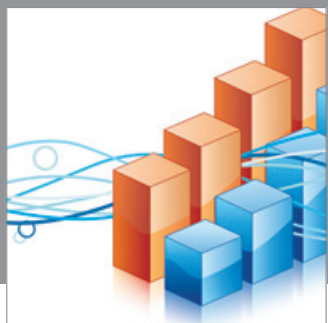

Advances in

Operations Research

mansans

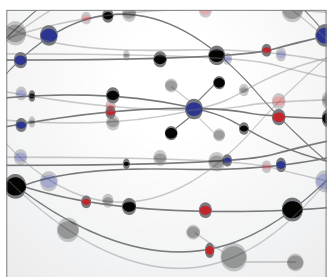

The Scientific World Journal
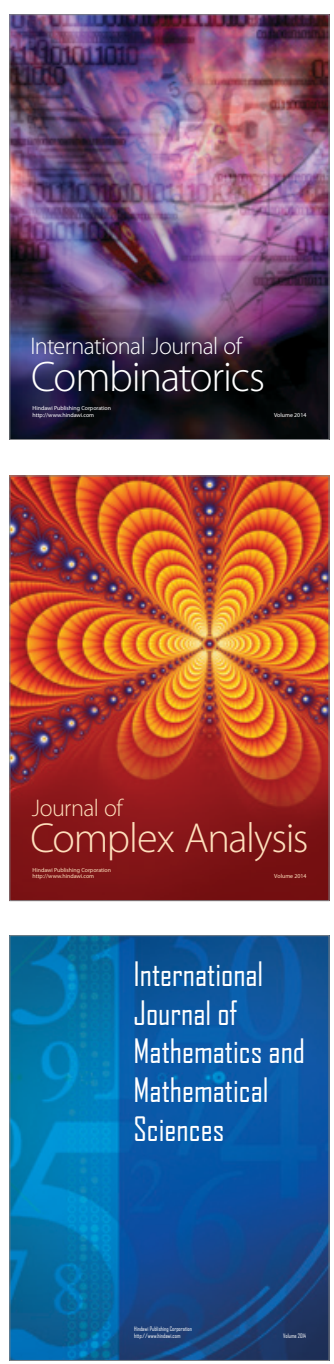
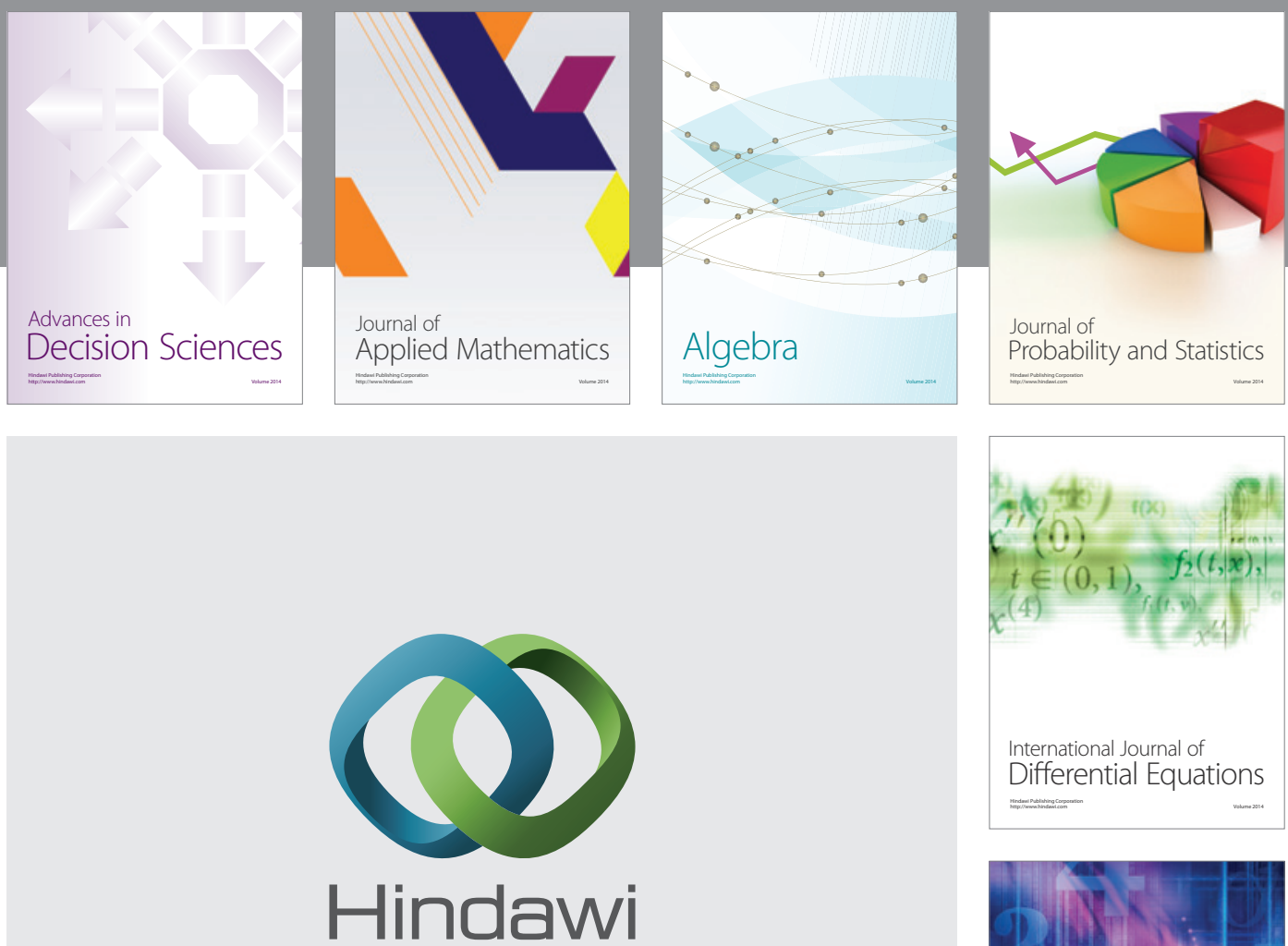

Submit your manuscripts at http://www.hindawi.com
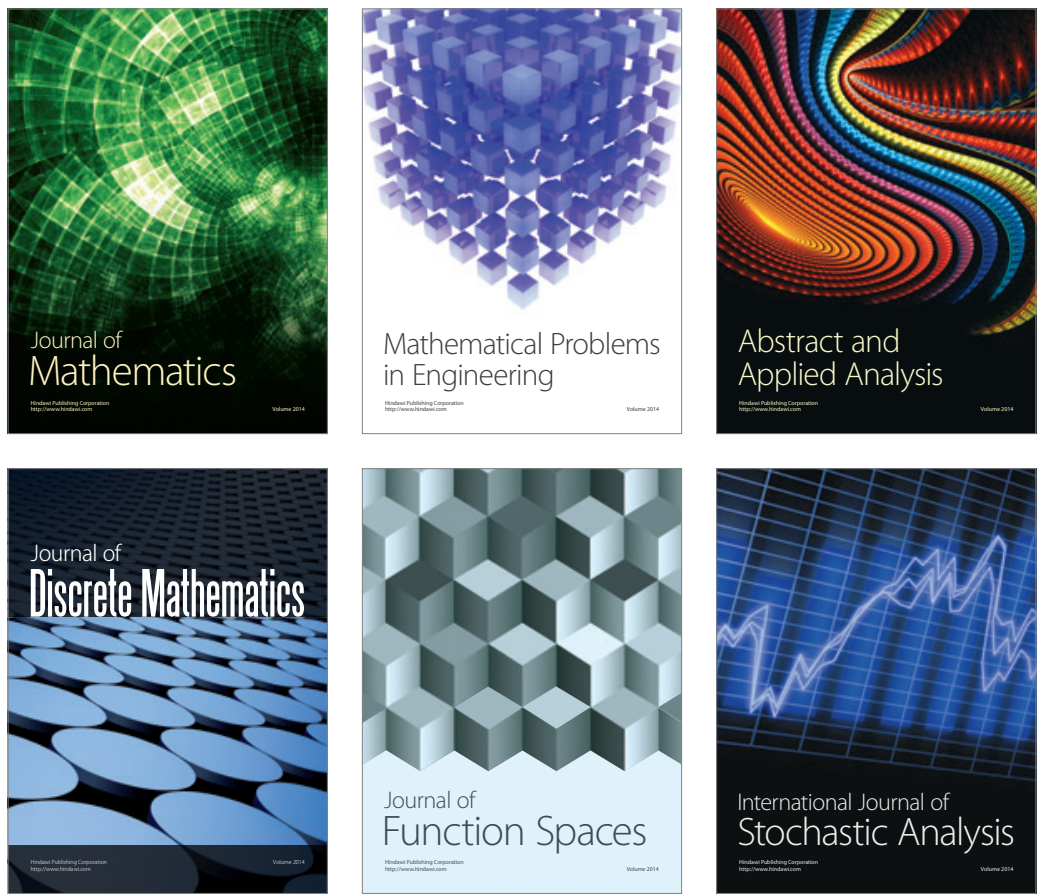

Journal of

Function Spaces

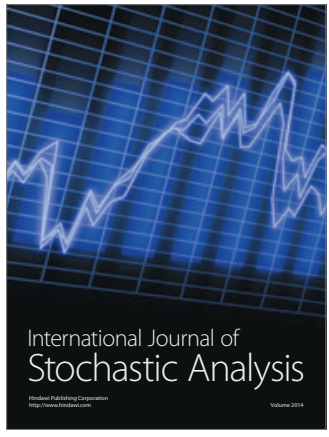

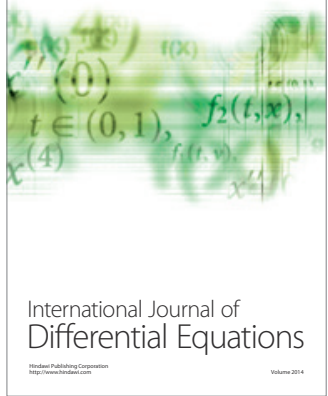
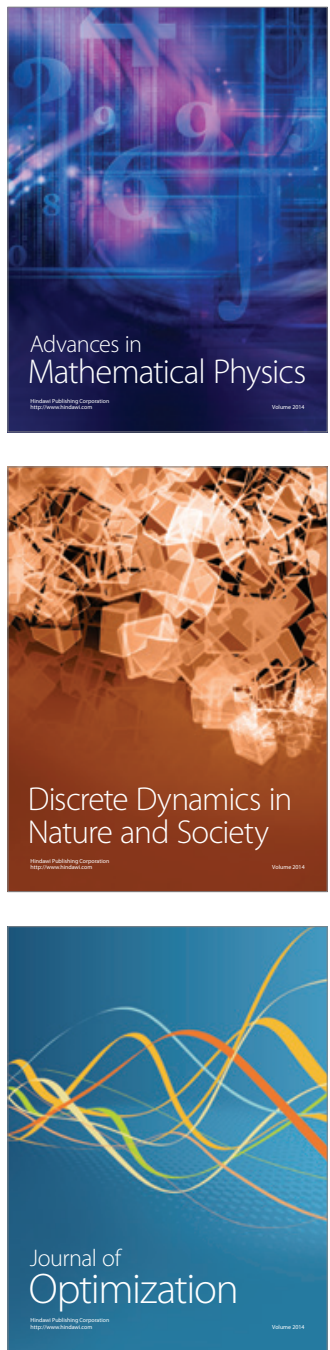